\title{
Two-part Allegorical Saying of Insect in Northeast Dialect and Its Cultural Implication
}

\author{
ShuanJun $\mathrm{An}^{1, *}$, SiYuan $\mathrm{Li}^{2}$

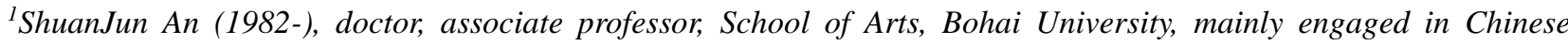 \\ phonology and Chinese dialectology. \\ ${ }^{2}$ SiYuan Li (1998-), studying for a master's degree, School of Arts, Bohai University, mainly engaged in the research of \\ teaching Chinese to speakers of other language. \\ *E-mail: anshuanjun123@126.com \\ ${ }^{2}$ E-mail: 1987720256@qq.com
}

\begin{abstract}
Two-part allegorical saying is a special linguistic form, which comes from the folk, reflecting the wisdom of the working people. There are abundant Two-part allegorical sayings of animal in the Northeast dialect, which are illustrated by the images or actions of animals and have good expression effect. This paper mainly introduces Two-part allegorical sayings of insect in Northeast dialect from the perspective of linguistics and culture, involving 19 kinds of insects. It is regarded as Two-part allegorical sayings of insect as long as the first part of Two-part allegorical sayings of insect is related to insects.
\end{abstract}

Keywords: Northeast dialect, Two-part allegorical sayings of insect, Cultural implication

\section{INTRODUCTION}

Two-part allegorical saying is a special linguistic form created by the working people in their daily life. It is characterized by short, concise, vivid and persuasive. There are many kinds of Two-part allegorical saying in Northeast dialect. The paper mainly introduces the Two-part allegorical saying related to insects. The first part of Two-part allegorical saying related to insects is regarded as Two-part allegorical sayings of insect. It mainly involves flea, louse, maggot, grasshopper, ant, fly, gadbee, firefly, scorpion, Atractomorpha sinensis bolvar, wasp, Apis cerana, bee, mole cricket, dung beetle, katydid, mosquito, cricket, bedbug and other nearly 19 kinds of insects. Biologically, the scorpion belongs to the class Arachnida in the phylum arthropoda, along with the class insect. In Northeast dialect, people used to call scorpion insect, so the "insects" in this article also includes scorpion. In Chinese, insects originally written as three insects, because it like to cluster together, as well as "three" represent a large number in Chinese literature. In Shuo Wen Jie $\mathrm{Zi}$ by $\mathrm{Xu}$ Shen, those with feet are called "insects and those without feet are called " $z h i$ ". The original meaning of "chong" is the meaning of bugs, insects, such as the words "insignificant skill (doing something as easy as an bug)", "beneficial bug", and then as a universal name for animals, such as some areas called the tiger "big bug", called the snake "long bug". In Chinese, the word "insects" read "hui", on oracle bone script it looks like a snake. In the specification, the word is identified as "chong". [1]

\section{NOMINAL PHRASES}

According to the structure of the first part of Two-part allegorical saying, it can be divided into nominal phrases and predicate phrases. Nominal phrases, also known as "nominal phrase" and "nominal structure". The overall grammatical function is consistent with nouns and other nominal words. Nominals are "nominal words". Nominal phrases include nouns, pronouns (pronouns and personal pronouns), numerals, quantifiers, and nominal phrases. For ease of understanding, "nominal words" or "noun" are sometimes used instead of "nominals" and "nominal phrases". Such as "hungry lice", "maggots in sauce jar", "scorpion shit", etc.

According to the description of the first part of Two-part allegorical saying, it can be divided into two categories: 


\subsection{The first part describes the insects in different situations, and the second part gives explanations}

"Flea" is called "jumping" or "dog insect" in Northeast dialect. It is a small, wingless, jumping parasitic insect that feeds on blood. The adult fleas usually live on humans, cats, dogs and other mammals, a few live on birds. "The fleas in the quilt -- where can they jump to?" It is a metaphor for someone's limited ability to achieve great things. Lice is also a parasitic insect, lice usually parasitic on humans or cats, dogs and other mammals, a few parasitic on marine mammals. "A louse on a bald head -- it's obvious", it is pun and metaphor that means something is easy to see. "Maggots in sauce jar -- salty (idle)", which has two meanings. One is meddlesome and the other is idle. "Salty" in Chinese pronunciation is homophonic with "idle". In the second part of the explanation, there is another saying that "Maggots in sauce jars -- salty (idle)", which literally means maggots wander around in jars with very salty sauce. The Chinese pronunciation of "salty" is "xian" that sounds similar to the Chinese pronunciation of "idle", which means wandering aimlessly at leisure. Locusts, commonly known as "grasshoppers", are called "flying grasshoppers" in some places in Northeast China. They like to eat corn, rice, beans, vegetables, fruit trees, weed leaves, etc. Many of these pests are found in the Northeast. After autumn, when the weather gets cooler, grasshoppers will die one after another. Therefore, people created the Two-part allegorical saying "Grasshoppers after autumn -- they can't hop for a few days" or "Grasshoppers at frost's descent -- hopping to the end", which is generally used for satire, means that things or undertakings cannot be sustained and face failure or destruction due to certain conditions. "Headless grasshopper -- hopping blindly", the grasshopper will not die immediately after losing its head and will collide indiscriminately because it is invision and pain. ${ }^{[2]}$ This sentence refers to people's reckless action and condemnation. "Like a cat on hot bricks -- cornered" is a metaphor for getting into trouble and difficult to leave it. As we all know, the twelve Chinese zodiac signs include twelve kinds of animals, for example, rat, ox, tiger, rabbit, dragon, snakes, horse, goat, monkeys, rooster, dogs and boar. Two-part allegorical saying has the pattern: The first half uses the structure of "One's zodiac sign is..." and the second half explains it. The animals behind the "One's zodiac sign is..." can be one of the twelve Chinese zodiac signs, such as "One's zodiac sign is rats -- can eat but can't take", "One's zodiac sign is rabbits -- timid", or they can not in twelve zodiac signs, such as "One's zodiac sign is owls -- happy in the night", "One's zodiac sign is mules -- unprecedented", "One's zodiac sign is porcupines -covered with thorns", or people, such as "One's zodiac sign is $L v b u$-- brave and resourceless", "One's zodiac sign is A dou -- can't hold up", and even inanimate, such as "One's zodiac sign is bean cake -- pressing up and down" and "One's zodiac sign is funnel - can't fill fully". The Northeast dialect also uses the Two-part allegorical sayings created by this model, such as "One's zodiac sign is blind midges -- biting on meat", "blind midges", that is gadbees, which are slightly larger than flies and are good at sucking the blood of cattle, horses and other livestock, which means deliberately speaking to other people's sore spot. ${ }^{[3]}$ "One's zodiac sign is hungry lice -be stung (stared)". The Chinese pronunciation "stung" is homonymous with "stared". It means that you still don't let go and keep pestering when you get benefits, in other words, it expressed that someone are never satisfied whatever they already have. The first half of this kind of Two-part allegorical sayings can also be indicated directly by nouns, such as "Hungry lice -- be stung(stared)."

\subsection{The first part describes a part of an insect or insect's possessions, and the second part explains it}

"The bott of a firefly -- not much light(quantity)". The tail of a firefly can emit fluorescence at night, so it is called a firefly. After all, it is small and has limited light. The Chinese pronunciation "light" is homonymous with "quantity", and the meaning is that the index quantity is less or the ability is limited metaphorically. "Scorpion tail -- needle (really) poison". The tail of scorpion is a poisonous gland, needle shaped. When encountering prey or enemies, scorpions will firstly clamp it with double pincers, and then stab the poisonous needle into the other's body and inject venom. "Needle" is homonymous with "really" in Chinese pronunciation, which is a metaphor for sinister and vicious. "Scorpion shit -- to poison (only) one feces (share)", which literally means that scorpion feces are poisonous and unique, "poison" is homonymous with "only", and "feces" is homonymous with "share" in Chinese, which describes being different or special. "Atractomorpha sinensis bolvar's eyes -- growing long", "Atractomorpha sinensis bolvar" generally refers to the short frontal locusts, with slender eyes, which means that the length has increased.

\section{PREDICATE PHRASES}

Predicate phrases are also called "predicate phrase" and "predicate structures". The overall grammatical function is consistent with verbs and other predicates. There are mainly verb phrases, adjective phrases, and some quantitative phrases.

Two-part allegorical saying of insect of Northeast dialect can be classified into two parts: 


\subsection{Expressing a certain reason with the action of insects}

Expressing a certain reason with the action of insects. It can also be divided into two categories. One is the active behavior of insects, that is, they can complete it independently, such as "Grasshoppers jumping around the beach in October -- evil insects who don't know death". Another is that human beings use the image of insects to make up events to explain things, such as "Atractomorpha sinensis bolvar wearing straw hat -sharp (traitor) head does not show".

"Grasshoppers jumping around the beach in October -- evil insects who don't know death". In the North, October has reached the late autumn that the weather has turned cool, and the life of the grasshopper is about to the end, however, it is still jumping happily. This is a metaphor that refers to a people face the danger or dilemma without knowing it. "The wasp teaches the apprentice -- be it (sting) so", "keep" is homonymous with "sting" in Chinese pronunciation, which means that the matter has been discussed, so do it. It can also be said that "Apis cerana teach bees to sting -- like this (sting)", which means that things should be done like this. "Lala gryllotalpa wears a top hat -- pretend to be an outdated gentleman", "lala gryllotalpa" means "mole cricket" that are like lives in the soil. It is good at digging caves in the soil, looking for food in the excavation process, and laying eggs and breeding in the caves. "Wearing a top hat" is a proper behavior of people, not owned by animals. It satirizes some people pretends to be an identity. It is clearly not qualified, but it is hard to feign to be a big man. "A scorpion falls into a hole of the stone saddle-quern -- one sting (folding) and one grinding", "sting" is homonymous with "folding" in Chinese, which is a pun. It takes the meaning of "torture", mostly refers to being hit mentally or physically due to illness and troubles. "Flies produce maggots -- exploit loopholes". Flies produce maggots mean that flies lay eggs. Flies often choose the gap in feces or garbage dumps. It's a metaphor for people like to take shortcuts. "Flies in confinement -- reporting grievances (holding maggots)", "reporting grievances" is homonymous with "holding maggots" in the reading of Chinese, which refers to suffering or feeling grievances. "Baba dung beetles wear flowers -- show off shamelessly", "baba dung beetles" are dung beetles, which feed on animal feces and are known as "scavengers in nature". "show off shamelessly" can also be called "show one's beauty shamelessful", which means ridiculing someone to boast or show that they are beautiful and capable, and also means that a person feels good about himself, but this is not the case. ${ }^{[4]}$ "Baba dung beetles move -- rolling balls". Dung beetles often push and roll dung into balls for transportation. Here, people swear by the characteristics of dung beetles rolling dung balls. "Rolling balls" means go away, which contains the tone of command and threat to make

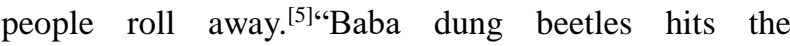
whirlwind foot -- passing the dung (degree)", "dung" is homonymous with "degree". Whirlwind foot is one of the four basic jumping movements of Chinese martial arts. It has the actions of flying and landing. "Passing the dung" originally means crossing the dung ball, which means exceeding a certain degree or limit. "Baba dung beetle takes a ship -- notorious" is a metaphor for being infamous and spreading a bad reputation far away. It is an ironic term.

\subsection{Insects, as a passive, are endowed with certain behaviors by people or other animals}

Insects, as a passive, are endowed with certain behaviors by people or other animals. Some behaviors are not facts and belong to human fictional events. Such as "Blind people catch katydids -- listening", "Blind bears catch ants --blindness busy", "Anti-aircraft shelling mosquitoes -- put fine timber to petty use".

"The blind bear catches the ant -- blindness busy", the ant is small, crawls fast and is not easy to catch. When the bear licks the ant, it is more laborious and difficult to fill his stomach. It's a Metaphor for aimless bustle. Derogatory language. "The blind bear catches the grasshopper -- fluttering blindly". The locust has wings and keeps jumping and flying, while the bear is clumsy and difficult to catch. It is a metaphor for futility, waste time and energy, and ineffective. Derogatory language. "The blind man catches the katydids -listening". The blind can't see anything, so he can only listen by ears to catch the katydids. "Listen" turns to remind others. "The toad catches the fly -- just enough to eat". For the toad, the fly is too small and not enough to fill the stomach. Originally it refers to a toad catches flies to eat just enough, and now the transfer meaning is that the income is just enough to live, and there is no surplus. ${ }^{[6] “ " M o n k e y ~ t a k e s ~ l i c e ~--~ n o n s e n s e ", ~ w h i c h ~ m e a n s ~}$ to unfounded nonsense speaking and fooling or futile. "Anti-aircraft shelling mosquitoes -- putting fine timber to petty use", which is a metaphor that says heroes have no place to play. ${ }^{[7]}$ "Demolishing the house to find crickets -- it's not worth it", which is a metaphor that means small gains outweigh big losses. "There is a bedbugs in the melon seed -- there are all kinds of nutlet (people)," "nutlet" is homonymous with "people" in Chinese pronunciation, which is a metaphor that portrays that all of characters are here so that people can't distinguish between true and false, good and bad, likes passing off fish eyes for pearls. ${ }^{[8]}$

\section{CONCLUSION}

The paper mainly introduces the Two-allegorical sayings about 19 kinds of insects, including flea, louse, maggot, grasshopper, ant, fly, gadbee, firefly, scorpion, 
Atractomorpha sinensis bolvar, wasp, Apis cerana, bee, mole cricket, dung beetle, katydid, mosquito, cricket, bedbug. These insects are commonly in Northeast China. According to the characteristics of insects, people explain things with the help of its images or actions, which reflects the wisdom of the working people. Firstly, according to the structural division of the first half of the Two-allegorical sayings, the Two-allegorical sayings of insect in Northeast dialect is divided into nominal phrases and predicate phrases. The nominal phrases are divided into two categories according to the description of the first half of the Two-allegorical sayings: Firstly, the half of Two-allegorical sayings describes insects in different situations, and the second half explains them; Secondly, the first part of the Two-allegorical sayings describes a certain part of the insect or insect property, and the second part explains it. Predicate phrases can be divided into two categories. One, expressing a certain reason with the help of insect action. Two, as a passive, insects are endowed with certain behaviors by people or other animals. Some behaviors are not facts and belong to human fictional events. When analyzing the Two-allegorical sayings, it focuses on the structure, meaning and cultural connotation of Two-allegorical sayings in this paper.

\section{Fund Project}

The key projects of social science planning fund of Liaoning Province "Investigation and research on dialects in the border area between Liaoning, Hebei and Inner Mongolia from the perspective of geolinguistics" (Project No: L19AYY005)

\section{REFERENCES}

[1] Wan Xianchu, Liu Huilong, Shuo Wen Jie Zi (12 Lectures) [M], Beijing: Zhonghua Book Company, 2019:109.

[2] Wang Shikai, Yang Liying, Northeast Dialect and Dulture $[\mathrm{M}]$, China International Broadcasting Press, 2014:35.

[3] Tang Yuwen, Northeast Dialect Dictionary [M], Changchun: Changchun Publishing House, 2012:365

[4] Yin Shichao, Northeast Dialect Concept Dictionary [M], Harbin: Heilongjiang University Press, 2010:89.

[5] Gao Yonglong, Northeast Dialect Dictionary [M], Beijing: Zhonghua Book Company, 2013:205.

[6] Zhao Hengyu, Huang Dianl, Charming Northeast Dialect [M], Shenyang: Liaoning Nationalities Publishing House, 2010:106.
[7] Dong Liansheng, Northeast Dialect of China [M] Hulunbuir: Inner Mongolia Culture Press, 2015:528.

[8] Zhang Guoyan, Notes on Jinzhou Dialect [M], Shenyang: Baishan Publishing House, 2013:217. 